\title{
Effects of RNAi-mediated MUC4 gene silencing on the proliferation and migration of human pancreatic carcinoma BxPC-3 cells
}

\author{
YONG LI $^{1 *}$, CHANGQIANG WU ${ }^{2 *}$, TIANWU CHEN ${ }^{1 *}$, JUANJUAN ZHANG $^{3}$, GANG LIU $^{4}$, YU PU $^{1}$, \\ JIANG ZHU ${ }^{5}$, CHENGYI SHEN ${ }^{6}$, YANG ZHANG ${ }^{1}$, NANLIN ZENG ${ }^{1}$ and XIAOMING ZHANG ${ }^{1}$
}

\begin{abstract}
${ }^{1}$ Sichuan Key Laboratory of Medical Imaging and Department of Radiology, Affiliated Hospital of North Sichuan Medical College, Nanchong, Sichuan 637000; ${ }^{2}$ Sichuan Key Laboratory of Medical Imaging and School of Medical Imaging, North Sichuan Medical College, Nanchong, Sichuan 637000; ${ }^{3}$ Sichuan Key Laboratory of Medical Imaging, North Sichuan Medical College, Nanchong, Sichuan 637000; ${ }^{4}$ State Key Laboratory of Molecular Vaccinology and Molecular Diagnostics and Center for Molecular Imaging and Translational Medicine, School of Public Health, Xiamen University, Xiamen, Fujian 361102; ${ }^{5}$ Sichuan Key Laboratory of Medical Imaging and Department of Chemistry, North Sichuan Medical College, Nanchong, Sichuan 637000; ${ }^{6}$ Sichuan Key Laboratory of Medical Imaging and Department of Pathophysiology, North Sichuan Medical College, Nanchong, Sichuan 637000, P.R. China
\end{abstract}

Received April 13, 2016; Accepted May 27, 2016

DOI: $10.3892 /$ or.2016.5152

\begin{abstract}
It was previously demonstrated that mucin 4 (MUC4) is not expressed in normal pancreatic tissues or in chronic pancreatitis tissue but is highly expressed in pancreatic cancer (PC) tissue. Effective MUC4 gene knockdown in PC may contribute to the elucidation of pancreatic tumor development and metastasis, and may be valuable in new therapeutic approaches. Thus to confirm this, in the present study, the BxPC-3 cell line was transfected with eight pairs of shRNA lentiviral vectors for MUC4. The qPCR results showed that expression of MUC4 mRNA in the BxPC-3 cells was significantly decreased at $96 \mathrm{~h}$ after transfection. One of these shRNA lentiviral vectors (shRNA-A141) had showed the strongest suppressive effect on MUC4 mRNA expression and was used for MUC4 knockdown in BxPC-3 cells. After stable transfection, BxPC-3 cells showed a significantly lower expression of MUC4 mRNA and MUC4 protein, and were suppressed on cell growth and migration. In vivo, lower tumor growth rates and tumor volume were observed in the tumors derived from the MUC4-knockdown cells, whereas the transplanted tumors derived from the control group cells,
\end{abstract}

Correspondence to: Professor Xiaoming Zhang, Sichuan Key Laboratory of Medical Imaging and Department of Radiology, Affiliated Hospital of North Sichuan Medical College, 63 Wenhua Road, Nanchong, Sichuan 637000, P.R. China

E-mail: zhangxm@nsmc.edu.cn; cjr.zhxm@vip.163.com

*Contributed equally

Key words: mucin, pancreatic carcinoma, RNA interference, lentivirus vectors grew rapidly. Thus, inhibition of MUC4 expression may be an effective means for mitigating metastasis and invasion of PC.

\section{Introduction}

The mortality rate of pancreatic cancer (PC) is high, and the 5-year survival rate is less than $6 \%$ in the United States (1-3) with an average survival period of 5 to 6 months. Thus, early diagnosis of PC is very important. PC cell metastasis negatively impacts radiation and chemotherapy $(4,5)$. The early diagnosis of PC is difficult due to a lack of clinical symptoms and reliable diagnostic markers. Previous studies have found aberrant overexpression of various mucins in various epithelial malignancies, including pancreatic and breast cancer (6-8). For example, mucin 1 (MUC1), MUC4 and MUC5AC are highly expressed in human $\mathrm{PC}$ tissues and are associated with disease progression and poor prognosis $(9,10)$. Using northern blot analysis and PCR, Andrianifahanana et al (11) found that MUC4 mRNA was not expressed in normal pancreatic tissues or in chronic pancreatitis tissue but was highly expressed in PC tissue. Therefore, MUC4 plays an important role in pancreatic tumor development and metastasis and may be a specific tumor marker for the diagnosis and therapy of PC $(12,13)$.

Mimeault et al found that silencing of the MUC4 gene can inhibit the growth and metastasis of PC (14). Effective MUC4 gene knockdown in PC has contributed to the elucidation of pancreatic tumor development and metastasis, and may be valuable in new therapeutic approaches. Recently, RNA interference (RNAi) has become widely used $(3,15-17)$ due to a more specific, effective and complete inhibition of gene expression compared to other methods. Short hairpin RNA (shRNA) for a specific gene can be integrated into the host chromosome using a lentivirus vector to stably express a small interfering RNA (siRNA), which can inhibit long-term 


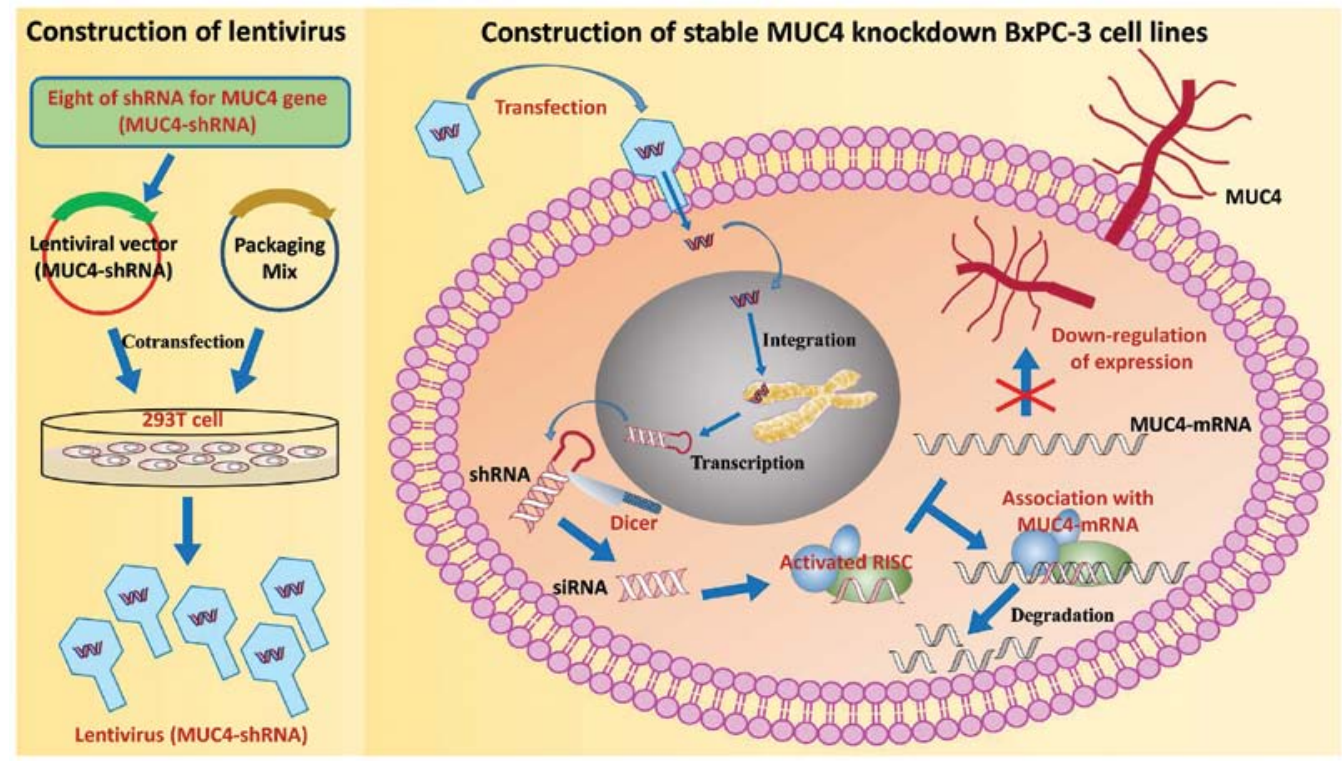

Figure 1. Eight shRNAs for the MUC4 gene (MUC4-shRNA) were designed and integrated into lentiviral vectors. The most efficient lentivirus for MUC4 mRNA interference was chosen for stable MUC4 knockdown in BxPC-3 cells.

gene expression. Here, we used BxPC-3 cells as an in vitro model of PC. Eight shRNAs for the MUC4 gene (MUC4shRNA) were designed and integrated into lentiviral vectors. The MUC4-shRNA with a high interference efficiency was identified and used for stable MUC4 knockdown in the BxPC-3 cell line (Fig. 1). Subsequently, cell proliferation, migration, protein expression, and tumor formation in vivo were assessed.

\section{Materials and methods}

Chemicals, reagents, and cell lines. The lentiviral expression vector was purchased from the Zimmer Medical International Trading Co., Ltd. (Shanghai, China). DH5a Escherichia coli (E. coli) was obtained from the Chinese Academy of Sciences (Shanghai, China). The restriction endonucleases BbsI and BamHI, T4 ligase and Taq enzyme were purchased from Takara (Dalian, China). Roswell Park Memorial Institute (RPMI)-1640 culture medium, fetal bovine serum (FBS), trypsin, Lipofectamine 2000 and TRIzol reagent were purchased from Gibco (Invitrogen Life Technologies, Carlsbad, CA, USA). A plasmid DNA extraction kit was obtained from Qiagen (Shanghai, China). A reverse transcription kit was purchased from Promega (Fitchburg, WI, USA). All oligonucleotides, including PCR primers, were purchased from Shanghai YingJun Biotechnology Co., Ltd. (Shanghai, China). A Cell Counting Kit-8 (CCK-8) was purchased from Dojindo Laboratories (Kumamoto, Japan). A Boyden chamber for the migration assay was obtained from Corning Costar (Rochester, NY, USA). Matrigel $(0.3 \mathrm{mg} / \mathrm{ml})$ was purchased from BD Biosciences (Bedford, MA, USA).

BxPC-3 cells, a human PC cell line, was purchased from the Shanghai Cell Bank of the Chinese Academy of Sciences. These cells were grown in RPMI-1640 media supplemented with $10 \%$ fetal calf serum, $100 \mathrm{U} / \mathrm{ml}$ penicillin and $100 \mu \mathrm{g} / \mathrm{ml}$ streptomycin at $37^{\circ} \mathrm{C}$ in a humidified atmosphere containing $95 \%$ air and $5 \% \mathrm{CO}_{2}$.
shRNA synthesis, vector construction and verification. The eight MUC4-siRNAs, and a negative control (with a scrambled sequence that did not match any known gene) were selected based on the full-length cDNA of human MUC4 mRNA (gene bank no. NM_004532) using Ambion siRNA design software. A siRNA against GAPDH was included as a positive control to verify transfection reliability, RNA extraction and gene expression quantification. The TTCAAGAGA sequence was used in the loop of all constructs to avoid premature termination. The sequences of eight shRNAs coresponding to MUC4-siRNA are listed in Table I. PCR products were annealed using the following conditions: $95^{\circ} \mathrm{C}$ for $5 \mathrm{~min}, 85^{\circ} \mathrm{C}$ for $4 \mathrm{~min}, 75^{\circ} \mathrm{C}$ for $5 \mathrm{~min}, 70^{\circ} \mathrm{C}$ for $5 \mathrm{~min}$ and preservation at $4^{\circ} \mathrm{C}$. After the annealing process, $20 \mu 1$ of shRNA template (10 $\mu \mathrm{l}$ of forward primer and $10 \mu \mathrm{l}$ of reverse primer), $10 \mu \mathrm{l}$ of annealing buffer (10X) and $70 \mu \mathrm{l}$ of double distilled water $\left(\mathrm{ddH}_{2} \mathrm{O}\right)$ were mixed at $100^{\circ} \mathrm{C}$ for ligation.

shRNA verification. After agarose electrophoresis purification, shRNA was inserted into the pUETP/td-tomoto/puro vector at the BamHI and BbsI sites. The resulting vector was inoculated into competent DH5 $\alpha$ E. coli, selected using kanamycin resistance, and verified with sequencing using Shanghai 3D Biopharm Bio-technology. A total of eight constructs, referred to as pUETP/td-tomoto/puro-shRNA-1-8 (A139-A146) were evaluated in cultured BxPC-3 cells. The experiment also included pUETP/td-tomoto/puro-shNC (shNC) as a negative control and pUETP/td-tomoto/puro-shPC (shPC) as a positive control.

Quantitative real-time PCR analysis ( $q P C R$ ). Two microliters of total RNA were reverse transcribed with Premix Ex Taq ${ }^{\mathrm{TM}}$ (Takara) using random primers. MUC4 first-strand cDNA was amplified with specific primers as follows: forward, 5'-GGAG AGGTATCGCCCTGATAGATT-3' and reverse, 5'-ACGGTA GTTGGGCCTTTCTTC-3'. The primers used for housekeeping gene, glyceraldehyde-phosphate dehydrogenase 
Table I. Sequences of the MUC4-shRNA primers.

No.

5'-3'-Primer sequences

\section{A139}

F GATCCGGCCTGTGAATTACTGCTACAATGGTACCATTGTAGCAGTAATTCACAGGTTTTTG

R AATTCAAAAACCTGTGAATTACTGCTACAATGGTACCATTGTAGCAGTAATTCACAGGCCG

A140

F GATCCGGCCCTGTGAATTACTGCTACAAGGTACCTTGTAGCAGTAATTCACAGGGTTTTTG

R AATTCAAAAACCCTGTGAATTACTGCTACAAGGTACCTTGTAGCAGTAATTCACAGGGCCG

A141

F GATCCGGGGGAACAACTTCAGTCCAACTGGTACCAGTTGGACTGAAGTTGTTCCCTTTTTG

A 142

R AATTCAAAAAGGGAACAACTTCAGTCCAACTGGTACCAGTTGGACTGAAGTTGTTCCCCCG

A142

F GATCCGGCAATGGTGGTCGTGTGATTGAGGTACCTCAATCACACGACCACCATTGTTTTTG

A143

R AATTCAAAAACAATGGTGGTCGTGTGATTGAGGTACCTCAATCACACGACCACCATTGCCG

F GATCCGGGCCCTGATAGATTCCTGAATTGGTACCAATTCAGGAATCTATCAGGGCTTTTTG

R AATTCAAAAAGCCCTGATAGATTCCTGAATTGGTACCAATTCAGGAATCTATCAGGGCCCG

A144

F GATCCGGCGCCCTGATAGATTCCTGAATGGTACCATTCAGGAATCTATCAGGGCGTTTTTG

R AATTCAAAAACGCCCTGATAGATTCCTGAATGGTACCATTCAGGAATCTATCAGGGCGCCG

A145

F GATCCGGAGTCCTGCCCTGTGAATTACTGGTACCAGTAATTCACAGGGCAGGACTTTTTTG

A146

R AATTCAAAAAAGTCCTGCCCTGTGAATTACTGGTACCAGTAATTCACAGGGCAGGACTCCG

F GATCCGGGGAGATGGCTATTTCGAAAACGGTACCGTTTTCGAAATAGCCATCTCCTTTTTG

R AATTCAAAAAGGAGATGGCTATTTCGAAAACGGTACCGTTTTCGAAATAGCCATCTCCCCG

F, forward; R, reverse.

(GAPDH), were as follows: forward, 5'-CATGAGAAGTATG ACAACAGCCT-3' and reverse, 5'-AGTCCTTCCACGATAC CAAAGT-3'. We evaluated the specificity of the primers using GenBank. The PCR products were separated on a $1.5 \%$ agarose gel and analyzed using an imaging system from Bio-Rad Laboratories, Inc. (Hercules, CA, USA).

Western blot (WB) analysis. Whole cell proteins were prepared from $5 \times 10^{6}$ cells after transfection for $96 \mathrm{~h}$ using WB lysis buffer. For WB, we used an anti-MUC4 mouse monoclonal antibody (8G7) and an HRP-linked anti-rabbit IgG secondary antibody (Santa Cruz Biotechnology, Inc., Santa Cruz, CA, USA). We used the BCA protein assay kit to detect the protein concentration in the supernatant (Takara, Otsu, Japan). The protein bands were visualized using an enhanced chemiluminescence system (Bio-Rad Laboratories).

Experiments in the BxPC-3 cells. After seeding in 6-well plates at a density of $1 \times 10^{5}$ cells/well, BxPC-3 cells were transfected with A139-A146, sh-NC, or sh-PC. After incubation for $96 \mathrm{~h}$, MUC4 mRNA was examined using qPCR. Subsequently, we counted the number of cells at 48,72 and $96 \mathrm{~h}$ after transfection using a colorimetric CCK-8 assay at $450 \mathrm{~nm}$. Migration was examined using a 24 -well, $8-\mu \mathrm{m}$ pore transparent insert containing a gelatin-coated polycarbonate membrane filter. Cell migration was quantified by counting the cells that migrated to the lower chamber using crystal violet staining and an optical microscope. Tumor formation and growth were examined in nude mice.

Statistical analyses. The quantitative results are expressed as the mean value \pm SD. All statistical analyses were carried out by one-way analysis of variance (ANOVA) using SPSS version 13.0 (SPSS, Inc., Chicago, IL, USA). A P-value of $<0.05$ was considered to indicate a statistically significant difference.

\section{Results}

Construction and verification of MUC4-shRNA lentiviral vector. Eight pairs of MUC4-shRNA were ligated into the lentiviral vector using T4 DNA ligase. A KpnI single enzyme digestion was performed, and the fragments were examined by agarose gel electrophoresis. A $2.95+7.5 \mathrm{~kb}$ fragment confirmed that the shRNA size conformed to the design (Fig. 2B). A transfection experiment on $293 \mathrm{~T}$ cells showed that more than $80 \%$ of the cells produced red fluorescent protein, as observed with fluorescence microscopy (Fig. 2D).

Construction of MUC4 knockdown in BxPC-3 cells. The $\mathrm{BxPC}-3$ cell lines were transfected with eight pairs of lentiviral vectors based on MUC4-shRNA. The qPCR results showed that expression of MUC4 mRNA in the BxPC-3 cells was significantly decreased by all eight shRNAs $(\mathrm{P}<0.05$ vs. the $\mathrm{NC}$; Fig. 3A) at $96 \mathrm{~h}$ after transfection. A141 had the strongest suppressive effect on MUC4 mRNA expression among the eight shRNAs and was used for further experiments. To obtain MUC4 knockdown in BxPC-3 cells, the BxPC-3 cell line was transfected with shRNA-A141, and cultured for nearly 20 days until the cell fluorescence ratio reached 100\% (Fig. 3B).

Proliferation, migration and protein expression of $M U C 4$ following knockdown in BxPC-3 cells. After stable transfection with shRNA-A141 (KD group), qPCR and western blot analysis were performed. The results showed that the efficiency of MUC4 mRNA interference was $91 \%$ (Fig. 4A). At the same time, MUC4 knockdown in the BXPC-3 cells (KD) showed 
A

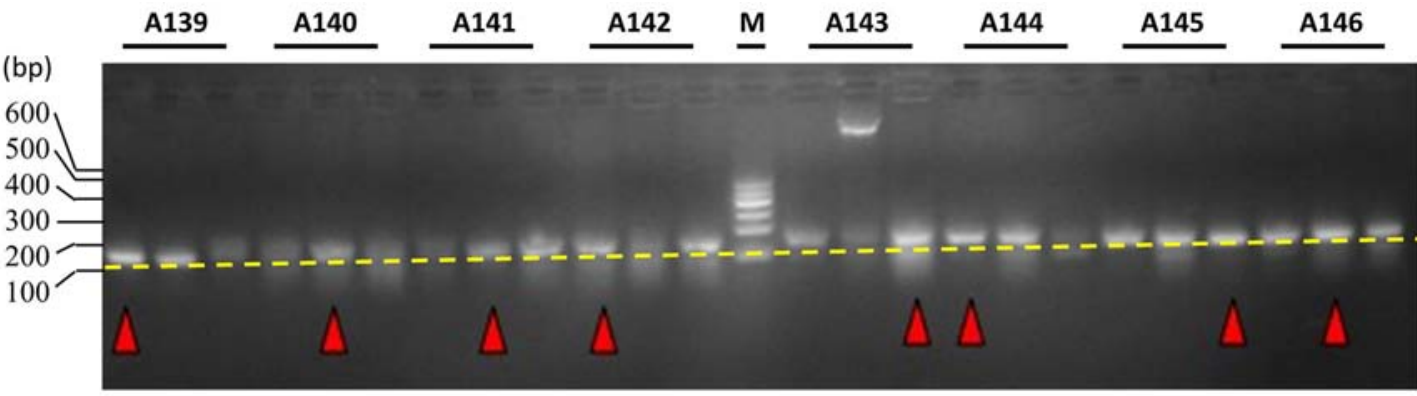

B
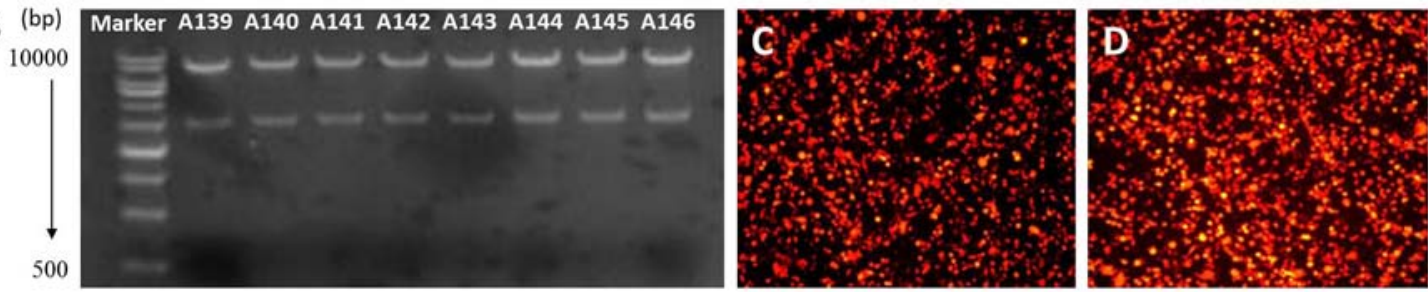

Figure 2. (A) Analysis by agarose gel electrophoresis of lentiviral vector (MUC4-shRNA) colony PCR. Positive clones contain a gene fragment of 170 bp. The marker size (M) ranged from 600 to 100 bp. (B) Agarose gel electrophoresis of lentiviral vector products after KpnI single enzyme restriction. Size fragments of 2.95 and $7.5 \mathrm{~kb}$ were observed, confirming that MUC4-shRNA was successfully integrated into the lentiviral vector. (C) Fluorescence microscopy of empty plasmid-transfected 293T cells. (D) Fluorescence microscopy of lentiviral vector (MUC4-shRNA-A141)-transfected 293T cells. More than 80\% of the cells produced red fluorescent protein. (Magnification, x100).

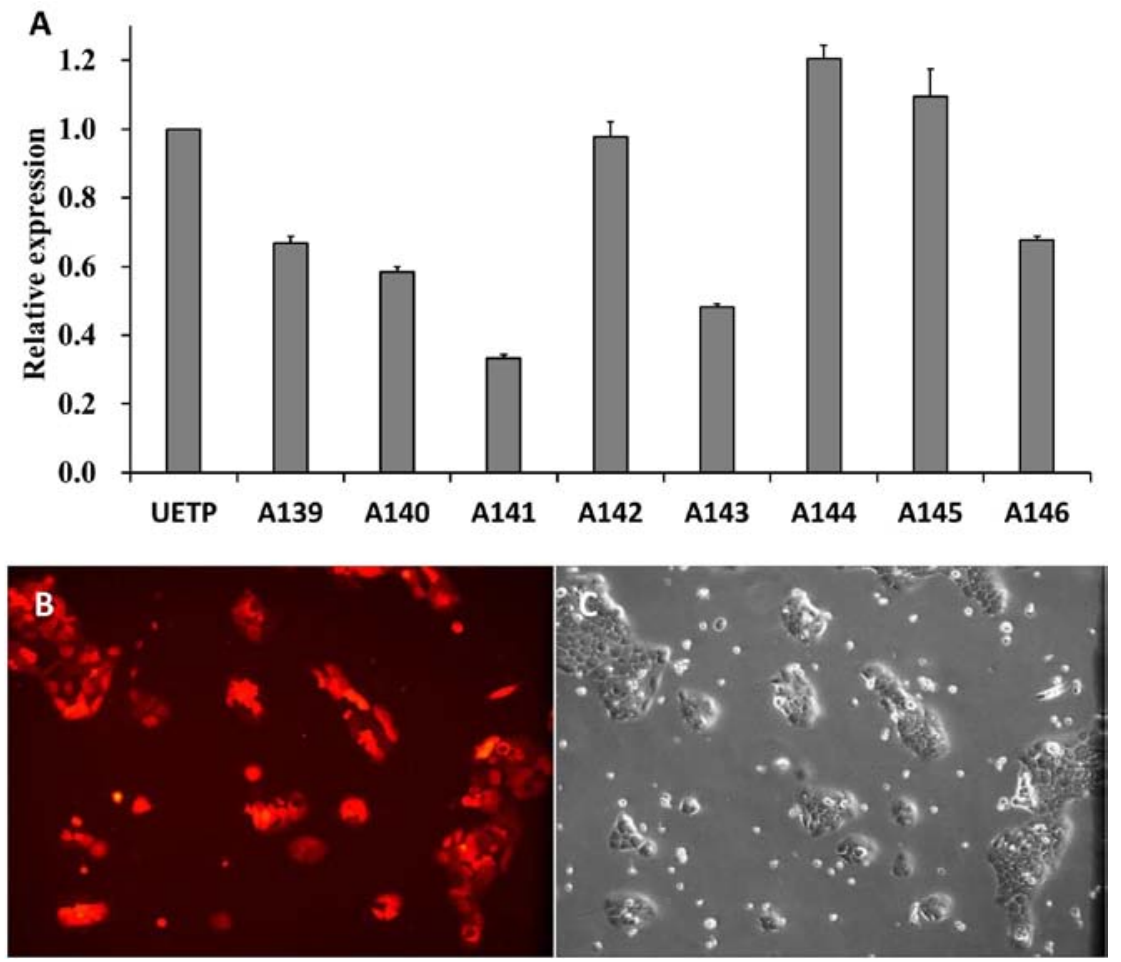

Figure 3. (A) MUC4 mRNA relative expression in BxPC-3 cells at $96 \mathrm{~h}$ after transfection with eight lentiviral vectors. A141 had the strongest suprressive effect on MUC4 mRNA expression. After stable transfection with recombinant lentivirus (A141 group), BxPC-3 cells were cultured for nearly 20 days, and observed under a fluorescence microscope system. (B) Fluorescent micrograph; (C) light micrograph. (Magnification, x200). Cell fluorescence reached 100\%.

reduced growth compared with cells transfected with empty or scrambled RNAi vectors (NC and CON groups), and the number of floating cells in the cell culture increased (Fig. 4B). Transwell migration assays showed that the number of cells in the polycarbonate membrane for the KD group were significantly lower than that of the NC group $(\mathrm{P}<0.01)$ (Fig. 4C). In fact, the expression level of MUC4 in the KD group was also significantly reduced, compared with the $\mathrm{NC}$ group $(\mathrm{P}<0.01)$ (Fig. 4D). The results showed that MUC4 protein knockdown in the BxPC-3 cells was noteworthy.

Tumor formation in vivo after MUC4 knockdown. BxPC-3 cells of the $\mathrm{KD}, \mathrm{NC}$ and $\mathrm{CON}$ groups were transplanted into nude mice. Tumors formed within 7-10 days. Lower tumor 

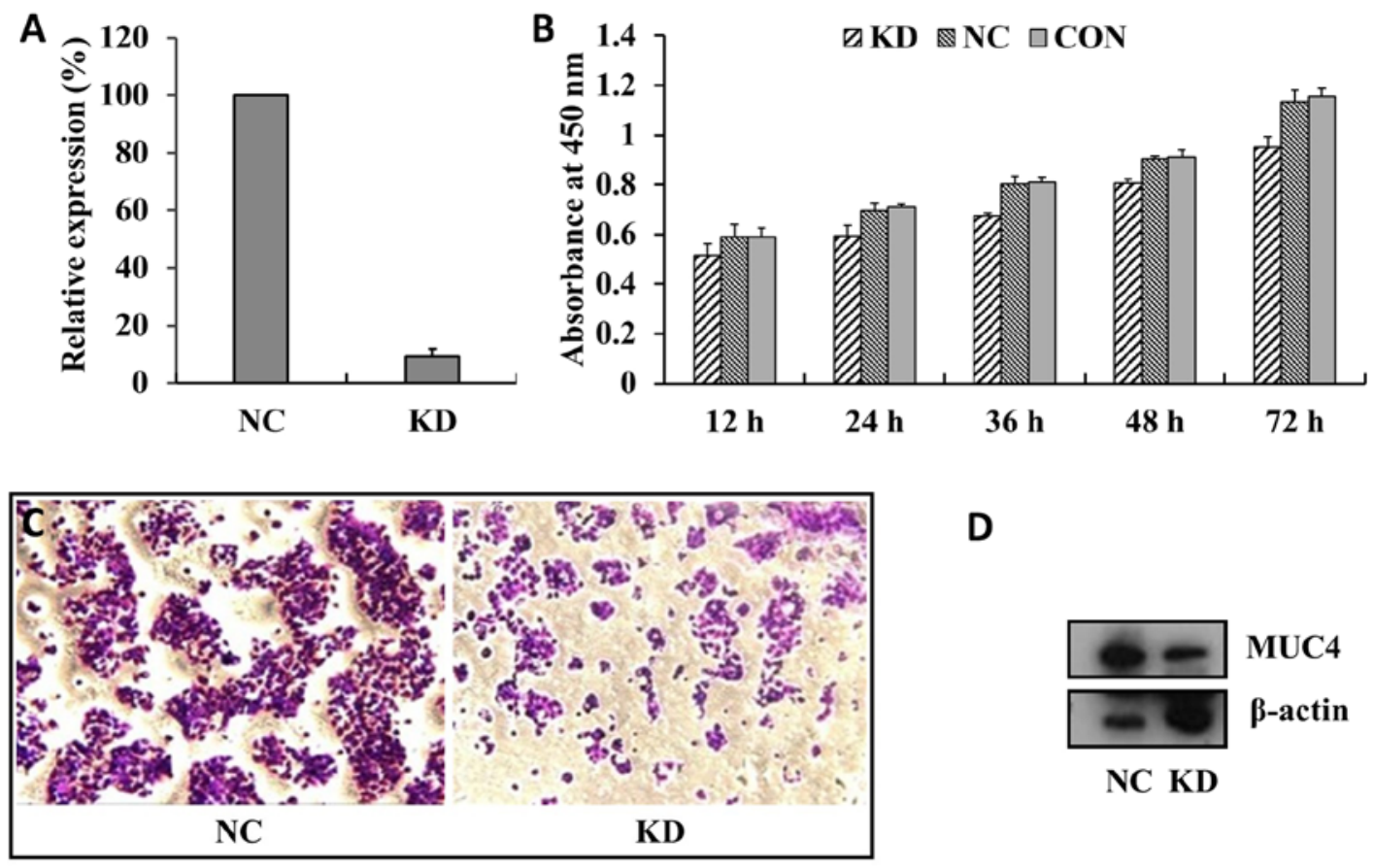

D

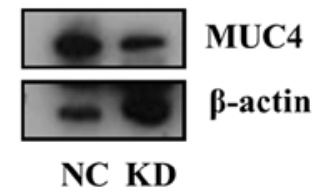

Figure 4 (A) Relative expression of MUC4 mRNA in the knockdown (KD) and negative control (NC) cells. The relative expression of MUC4 mRNA in the KD group was $\sim 9 \%$ and was significantly lower than that of the NC group. (B) Cell growth as measured in a CCK-8 assay. The cell growth rate of the KD group was significantly lower than the rate of the other groups. (C) Transwell migration assays. The cell migration in the KD group was significantly lower than the migration of the NC group. (D) MUC4 expression in the NC and KD BxPC-3 cells. MUC4 expression in the KD group was also decreased. CON, control group (BxPC-3). NC, empty plasmid transfection group (transfection with UETP); KD, MUC4 gene silencing group (stable transfection with shRNA-A141).

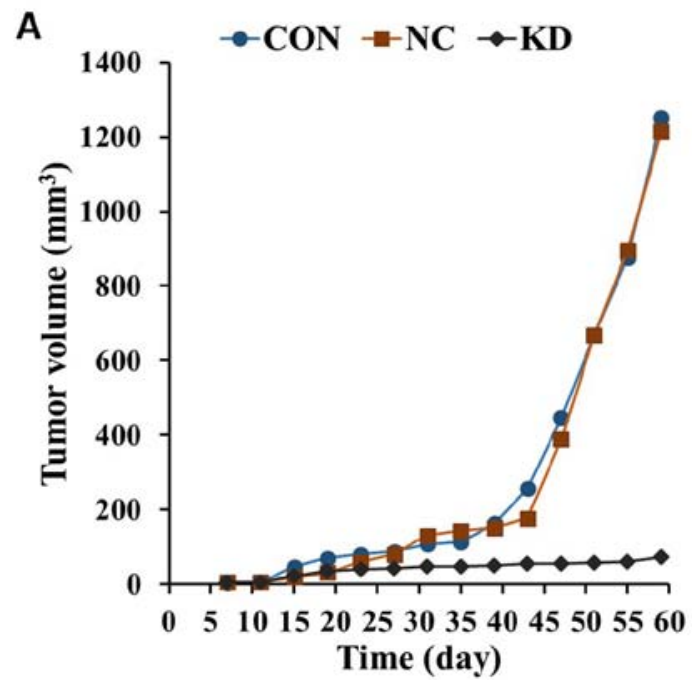

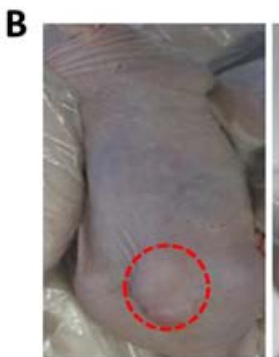

CON

C

\begin{tabular}{cc}
\hline Groups & Tumor volume $\left(\mathrm{mm}^{3}\right)$ \\
\hline $\mathrm{KD}$ & $195.02 \pm 14.24$ \\
$\mathrm{NC}$ & $2208.6 \pm 170.51$ \\
CON & $2123.98 \pm 127.81$ \\
\hline
\end{tabular}

Figure 5. BxPC-3 cell growth in nude mice. (A) The tumor growth-curve of the tumor volume across time for the three groups of nude mice. The tumor growth in the KD group was much slower than the growth in the other two groups. Compared with the CON group, $\mathrm{P}<0.01$ (KD group), $\mathrm{P}>0.05$ (NC group). (B) Images of tumor-bearing nude mice 50 days after cell inoculation. (C) Tumor volume measurements at 8 weeks after cell inoculation. CON, control group (BxPC-3); $\mathrm{NC}$, empty plasmid transfection group (transfection with UETP); KD, MUC4 gene silencing group (stable transfection with shRNA-A141).

growth rates were observed for tumors derived from the KD group, whereas the transplanted tumors derived from the other groups grew rapidly (Fig. 5A). At 8 weeks after cell inoculation, the tumor volume reached $195.02 \pm 14.24 \mathrm{~mm}^{2}$ in the $\mathrm{KD}$ group, which was less than a tenth of that in the $\mathrm{NC}$ and $\mathrm{CON}$ groups (Fig. 5C). Throughout the experiment, no significant differences were observed in the activity and eating behavior of the KD cell-transplanted nude mice. In the NC and CON groups, the activity of the nude mice gradually reduced with tumor enlargement.

\section{Discussion}

In the present study, MUC4 gene sequences of human PC BxPC-3 cells were used to design eight shRNAs (Table I). Typically, shRNAs are designed based on the principle that 
the first base in the shRNA sequence must be a G. If the first base is not a $\mathrm{G}$, a $\mathrm{G}$ must be added to the sense strand to ensure transcription by RNA polymerase. Furthermore, 5-6 Ts must be included at the $3^{\prime}$ end of the shRNA to ensure termination by RNA polymerase. However, more than 3 As or 3 Ts must appear in the sense strand and antisense strand to avoid early termination of shRNA transcription. Restriction enzyme sites must be at the ends of the two complementary nucleotide sequences, and the GC content should be between 36 and $52 \%$.

A lentiviral vector carrying the MUC4 gene was successfully constructed and eight pairs of shRNA were successfully ligated into the lentiviral vector pUETP. Positive clones were identified and chosen using colony PCR (Fig. 2A). PCR positive products had 2.95 and $7.5 \mathrm{~kb}$ fragments, as determined by agarose gel electrophoresis, after KpnI single enzyme digestion (Fig. 2B). Recombinant lentiviral vector plasmid, auxiliary plasmid and transit transfection reagents were transfected into the $293 \mathrm{~T}$ cells. More than $80 \%$ of the cells produced red fluorescent protein, as determined by fluorescence microscopy, which confirmed that the lentiviral vectors were efficiently transferred into the $293 \mathrm{~T}$ cells (Fig. 2D). Moreover, the viral titer was measured to confirm the lentiviral vector concentration necessary for subsequent experiments.

The expression of MUC4 mRNA in BxPC-3 cells was evaluated directly before and $96 \mathrm{~h}$ after transfection with the eight pairs of lentiviral vectors and was used to evaluate the interference efficiency. qPCR, as performed previously, was also used to analyze the interference efficiency $(18,19)$. The results showed that MUC4 mRNA in the A141-transfected group (MUC4-shRNA transfection KD group) had a significantly lower expression level when compared with A139, A140, A142, A143, A144, A145, A146 and the UETP groups. After stable transfection with shRNA-A141 in the BxPC-3 cells, the MUC4 mRNA knockdown was $\sim 91 \%$ (Fig. 4A, $\mathrm{P}<0.01$ ), which was significantly higher than that found in other studies $(20,21)$. These results suggest that our shRNAs designed against the MUC4 gene sequences effectively blocked MUC4 gene expression in the human PC BxPC-3 cells. Furthermore, MUC4 protein expression was detected by western blot analysis in the NC (UETP) and KD (A141) groups. MUC4 protein expression in the KD group was significantly reduced compared with the $\mathrm{NC}$ group $(\mathrm{P}<0.01)$. Our knockdown of MUC4 protein expression in the BxPC-3 cells was marked.

MUC4 is a high-molecular-weight glycol protein related to the growth, metastasis and angiogenesis of PC (21-24). Thus, we examined the cell growth and active state of the BxPC-3 cells in our study (Figs. 4 and 5). Compared with the NC group and CON group, the KD group had a lower cell growth rate and an increased number of floating cells. These results are consistent with other studies $(14,20)$ suggesting that the MUC4-shRNA used in our study could promote the apoptosis of BxPC-3 cells. Moreover, we also found that cell proliferation in the KD group was significantly suppressed compared with the proliferation in the $\mathrm{NC}$ and CON groups, as determined by a CCK-8 cell proliferation test. We found that cell migration in the KD group was significantly lower than the migration in the NC group, as determined using a Transwell chamber test. These results are similar to previous results showing that MUC4 knockdown reduces the migration ability of PC cells (24) Moreover, BxPC-3 cells of the KD, NC and $\mathrm{CON}$ groups were transplanted into nude mice. Lower tumor growth rates were observed for tumors derived from the KD group, whereas the transplanted tumors derived from the other groups grew rapidly (Fig. 5). At 8 weeks after cell inoculation, the tumor volume in the KD group was less than a tenth of that in the NC and CON groups.

In conclusion, we demonstrated for the first time that MUC4 promotes the migration and proliferation of $\mathrm{BxPC}-3$ cells. These results indicate that inhibition of MUC4 expression may be an effective means for limiting metastasis and invasion of PC cells. Thus, MUC4 may be a new potential target for the treatment of PC.

\section{Acknowledgments}

This study was supported by the National Natural Science Foundation of China, no. 81271643.

\section{References}

1. Siegel R, Naishadham D and Jemal A: Cancer statistics, 2012. CA Cancer J Clin 62: 10-29, 2012.

2. Frank TS, Sun X, Zhang Y, Yang J, Fisher WE, Gingras MC and Li M: Genomic profiling guides the choice of molecular targeted therapy of PC. Cancer Lett 363: 1-6, 2015.

3. Rachagani S, Macha MA, Heimann N, Seshacharyulu P, Haridas D, Chugh S and Batra SK: Clinical implications of miRNAs in the pathogenesis, diagnosis and therapy of PC. Adv Drug Deliv Rev 81: 16-33, 2015.

4. Matsuno S, Egawa S, Fukuyama S, Motoi F, Sunamura M, Isaji S, Imaizumi T, Okada S, Kato H, Suda K, et al: PC Registry in Japan: 20 years of experience. Pancreas 28: 219-230, 2004.

5. Sultana A, Tudur Smith C, Cunningham D, Starling N, Neoptolemos JP and Ghaneh P: Meta-analyses of chemotherapy for locally advanced and metastatic PC: Results of secondary end points analyses. Br J Cancer 99: 6-13, 2008.

6. Kufe DW: Mucins in cancer: Function, prognosis and therapy. Nat Rev Cancer 9: 874-885, 2009.

7. Ponnusamy MP, Seshacharyulu P, Lakshmanan I, Vaz AP, Chugh S and Batra SK: Emerging role of mucins in epithelial to mesenchymal transition. Curr Cancer Drug Targets 13: 945-956, 2013

8. Kaur S, Kumar S, Momi N, Sasson AR and Batra SK: Mucins in $\mathrm{PC}$ and its microenvironment. Nat Rev Gastroenterol Hepatol 10: 607-620, 2013.

9. Torres MP, Chakraborty S, Souchek J and Batra SK: Mucin-based targeted PC therapy. Curr Pharm Des 18: 2472-2481, 2012.

10. Rachagani S, Torres MP, Kumar S, Haridas D, Baine M, Macha MA, Kaur S, Ponnusamy MP, Dey P, Seshacharyulu P, et al: Mucin (Muc) expression during PC progression in spontaneous mouse model: Potential implications for diagnosis and therapy. J Hematol Oncol 5: 68, 2012.

11. Andrianifahanana M, Moniaux N, Schmied BM, Ringel J, Friess H, Hollingsworth MA, Büchler MW, Aubert JP and Batra SK: Mucin $(M U C)$ gene expression in human pancreatic adenocarcinoma and chronic pancreatitis: a potential role of MUC4 as a tumor marker of diagnostic significance. Clin Cancer Res 7: 4033-4040, 2001.

12. Wu SC, Chen YJ, Lin YJ, Wu TH and Wang YM: Development of a mucin4-targeting SPIO contrast agent for effective detection of pancreatic tumor cells in vitro and in vivo. J Med Chem 56: 9100-9109, 2013.

13. Jhala N, Jhala D, Vickers SM, Eltoum I, Batra SK, Manne U, Eloubeidi M, Jones JJ and Grizzle WE: Biomarkers in diagnosis of pancreatic carcinoma in fine-needle aspirates: A translational research application. Am J Clin Pathol 126: 572-579, 2006.

14. Mimeault M, Johansson SL, Senapati S, Momi N, Chakraborty S and Batra SK: MUC4 down-regulation reverses chemoresistance of PC stem/progenitor cells and their progenies. Cancer Lett 295: 69-84, 2010.

15. Ambesajir A, Kaushik A, Kaushik JJ and Petros ST: RNA interference: A futuristic tool and its therapeutic applications. Saudi J Biol Sci 19: 395-403, 2012. 
16. Pecot CV, Calin GA, Coleman RL, Lopez-Berestein G and Sood AK: RNA interference in the clinic: Challenges and future directions. Nat Rev Cancer 11: 59-67, 2011.

17. Agrawal N, Dasaradhi PV, Mohmmed A, Malhotra P, Bhatnagar RK and Mukherjee SK: RNA interference: Biology, mechanism, and applications. Microbiol Mol Biol Rev 67: 657-685, 2003

18. Zhang C, Fu J, Wang Y, Bao Z and Zhao H: Identification of suitable reference genes for gene expression normalization in the quantitative real-time PCR analysis of sweet osmanthus (Osmanthus fragrans Lour.). PLoS One 10: e0136355, 2015.

19. Dijkstra JR, van Kempen LC, Nagtegaal ID and Bustin SA: Critical appraisal of quantitative PCR results in colorectal cancer research: Can we rely on published qPCR results? Mol Oncol 8: 813-818, 2014

20. Chaturvedi P, Singh AP, Moniaux N, Senapati S, Chakraborty S, Meza JL and Batra SK: MUC4 mucin potentiates pancreatic tumor cell proliferation, survival, and invasive properties and interferes with its interaction to extracellular matrix proteins. Mol Cancer Res 5: 309-320, 2007.
21. Singh AP, Moniaux N, Chauhan SC, Meza JL and Batra SK: Inhibition of MUC4 expression suppresses pancreatic tumor cell growth and metastasis. Cancer Res 64: 622-630, 2004

22. Zhi X, Tao J, Xie K, Zhu Y, Li Z, Tang J, Wang W, Xu H, Zhang J and Xu Z: MUC4-induced nuclear translocation of $\beta$-catenin: A novel mechanism for growth, metastasis and angiogenesis in PC. Cancer Lett 346: 104-113, 2014.

23. Rachagani S, Macha MA, Ponnusamy MP, Haridas D, Kaur S, Jain M and Batra SK: MUC4 potentiates invasion and metastasis of PC cells through stabilization of fibroblast growth factor receptor 1. Carcinogenesis 33: 1953-1964, 2012.

24. Lahdaoui F, Delpu Y, Vincent A, Renaud F, Messager M, Duchêne B, Leteurtre E, Mariette C, Torrisani J, Jonckheere N, et al: $\mathrm{miR}-219-1-3 \mathrm{p}$ is a negative regulator of the mucin MUC4 expression and is a tumor suppressor in PC. Oncogene 34: 780-788, 2015. 